finding. Positive findings included microcolon, volvulus, Hirschprungs and obstruction. The majority of infants were discharged with no complications.

Discussion Bilious vomiting is synonymous with intestinal obstruction and should be considered this until proven otherwise . Management is time critical given the potential consequences of a volvulus and the ischaemic threat to the bowel with the window of opportunity being approximately six hours (3) A national guideline is now warranted.

\section{P476 SIRENOMELIA (MERMAID SYNDROME) - A RARE CONGENITAL DISORDER}

${ }^{1}$ Muhammad Imran Riazat*, 'Jamaleddin Abujennah, ${ }^{2}$ Bharti Kewlani, ${ }^{1}$ Farhana Sharif. ${ }^{1}$ Mullingar Regional Hospital, Mullingar, Ireland; ${ }^{2} R C S I$, Dublin, Ireland

10.1136/archdischild-2019-epa.812

Background Sirenomelia or mermaid syndrome is an extremely rare congenital disorder involving the lower spine and lower limbs. Although usually fatal in the newborn period, survival in a handful of cases beyond infancy have been reported. We would like to present a new born with sirenomelia and multiple anomalies brought in to our hospital soon after birth.

Case report A 40 year old Romanian lady G 17, P 16, Ab 0, known diabetic poorly controlled on insulin, delivered at 38 weeks gestation in an ambulance en route to the hospital. On arrival, she was taken directly to the labor ward with ongoing resuscitation efforts with bag and mask and CPR carried out by the paramedics. The baby was noted to be cyanosed and in respiratory distress. Severe congenital abnormalities were obvious and included flattened dysmorphic features, low set malformed ears, upward slanting palpebral fissures, flattened nose, receding chin, short neck, small thoracic cage, ambiguous genitalia, absent anal opening and fused lower limbs. The feet however, were separate.

The mother was originally booked in a tertiary referral center where she had undergone antenatal scanning and was counselled on the poor prognosis due to multiple abnormalities detected including but not limited to anhydramnios (that could result in pulmonary hypoplasia), absent left kidney, right cystic dysplastic kidney and cardiac malformation. When the mother was brought in by ambulance into our hospital, we were not aware of the management plan. The baby was therefore intubated and started on positive pressure ventilation as per the protocol. Shortly after, we realized futility after resuscitation became obvious. We received more information regarding the poor prognosis and consequently, extubated the baby at 18 minutes of life and handed over the baby to the mother for comfort care.

Genetic blood tests for microarray and karotyping were carried out and revealed female karyotype with normal microarray. Her skeletal survey was requested which showed caudal regression and absence of vertebras after S1, malformed pelvis and soft tissue fusion of both lower limbs, however feet were separated.

Conclusion Sirenomelia is an extremely rare and usually fatal congenital malformation. Maternal diabetes, smoking and heavy metal exposure have been implicated as possible etiologies. Therefore, in our opinion poor glycemic control prior to conception and during gestation (especially during the first trimester) could have contributed to this condition.

\section{P477} TWO CASES OF LATE ONSET GBS INFECTION DESPITE POST NATAL BENZYLPENICILLIN ADMINISTRATION

${ }^{1}$ Simon Piggott*, 'Edina Moylett, 'Ethel Ryan, ${ }^{2}$ Una Ni Riain. ${ }^{1}$ Academic Department of Paediatrics, National University of Ireland, Galway, Galway, Ireland; 'Department of Microbiology, University Hospital Galway, Galway, Galway, Ireland

\subsection{6/archdischild-2019-epa.813}

Aim Streptococcus agalactiae (Group B streptococcus, GBS) is the leading cause of invasive infection among neonates and young infants in the developed world. The prevention of late onset GBS disease (LOD) remains elusive. We present two cases of LOD, one near fatal with significant morbidity, despite early treatment with benzylpenicillin in each case.

Method Following confirmation of GBS LOD, the medical record for each infant was reviewed at University Hospital Galway. Relevant clinical and laboratory information was recorded.

Results Case 1, following an intrapartum high vaginal swab positive for GBS with a clinical suspicion for chorioamnionitis at time of delivery, an otherwise well infant (born at $28+6$ weeks) was treated empirically with a 5 day course of IV benzylpenicillin and gentamicin despite sterile blood cultures. The infant's neonatal intensive care unit (NICU) course was uneventful until day of life (DOL) 25 when following a clinical deterioration necessitating a sepsis evaluation, the infant's blood culture isolated GBS, the infant made a full recovery. Case 2, a singleton female infant (born at $31+6$ weeks) was treated empirically with a 48 hour course of IV benzylpenicillin and gentamicin owing to an intra-uterine death of unknown cause at 18 weeks during a triplet pregnancy. There were no positive swabs/clinical suspicion for intra-partum infection with neonatal sterile blood cultures following delivery. Standard NICU care was carried out until DOL 9 when the infant developed overwhelming sepsis with GBS positive blood cultures and was transferred to a tertiary NICU centre for further care. The infant survived with significant morbidities. In both of our cases, multi-locus sequence typing and serotyping allowed accurate identification of isolates. Our chosen cases demonstrate the continued prevalence of GBS and the recognised limitations of prophylactic antibiotic use with both of the infants developing GBS infection between days 7-89 of life despite initial antibiotic administration.

Conclusion Antenatal risk-based guidelines and antibiotic prophylaxis are effective in ameliorating early onset GBS infection but are inadequate for combating LOD. Admittedly, the pathogenesis of LOD remains elusive and the route of acquisition is still unclear, possibly vertically from mother or horizontally from environmental sources. Our cases support each of these theories. Postnatal antibiotic therapy does not protect against LOD highlighting the potential role for vaccination in LOD prevention.

\section{P478 RARE CASE OF SEVERE THROMBOCYTOPENIA AT BIRTH ASSOCIATED WITH RHESUS DISEASE OF THE NEWBORN}

${ }^{1}$ Alina G Deliu*, Dr Melany Cotter ${ }^{2}$, Dr ${ }^{3}$ JB Letshwiti. 'Mayo University Hospital , Castlebar, Ireland; '2Our Lady's Children's Hospital, Crumlin , Dublin, Ireland; ${ }^{3}$ Galway University Hospital, Galway, Ireland

\subsection{6/archdischild-2019-epa.814}

Aims Rhesus isoimmunization (RI) is becoming relatively uncommon with the introduction of Rho (D) immune globulin 